\section{PSICOLOGIA IBEROAMERICANA}

\section{Psicología lberoamericana}

ISSN: 1405-0943

psicología.iberoamericana@uia.mx

Universidad Iberoamericana, Ciudad de

México

México

Trejo Pérez, Fabiola; Díaz-Loving, Rolando

En torno a la sexualidad: actitudes y orientación sociosexual en una muestra mexicana

Psicología Iberoamericana, vol. 21, núm. 1, enero-junio, 2013, pp. 7-15

Universidad Iberoamericana, Ciudad de México

Distrito Federal, México

Disponible en: http://www.redalyc.org/articulo.oa?id=133929862002

Cómo citar el artículo

- Número completo

- Más información del artículo

- Página de la revista en redalyc.org

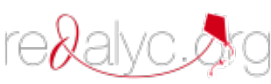

Sistema de Información Científica

Red de Revistas Científicas de América Latina, el Caribe, España y Portugal

Proyecto académico sin fines de lucro, desarrollado bajo la iniciativa de acceso abierto 


\title{
En torno a la sexualidad: actitudes y orientación sociosexual en una muestra mexicana
}

\author{
In Regards to Sexuality: Attitudes and Sociosexual Orientation \\ in a Mexican Sample
}

\author{
Fabiola Trejo Pérez \\ Rolando Díaz-Loving* \\ UnIDAD de InVESTIGACIONES PSICOSOCIALES \\ Universidad Nacional Autónoma de México
}

\section{RESUMEN}

Para sociedades conservadoras como la mexicana, la sexualidad y las relaciones de pareja se encuentran estrechamente vinculadas. Dicho contexto sociocultural facilita la construcción de gran variedad de actitudes que determinan el nivel de restricción o permisividad de una persona para involucrarse en relaciones sexuales. En el presente estudio se hace un análisis de las actitudes y comportamientos ante la sexualidad que manifiestan 112 jóvenes mexicanos mediante la aplicación del Inventario de Orientación Sociosexual $\alpha=.774$ y la escala de actitudes hacia la sexualidad $\alpha=.736$. Los resultados manifiestan poca apertura a involucrarse en actividades sexuales sin compromiso, con una tendencia moderada hacia la permisividad para establecer relaciones a corto plazo disociadas del afecto y compromiso, enfocadas en la actividad sexual. Aun con los cambios socioculturales en materia de sexualidad, es posible observar el impacto de la cultura tradicional y conservadora sobre las actitudes y comportamientos de los jóvenes.

Palabras clave: orientación sociosexual, actitudes, sexualidad, relaciones sexuales, pareja.

\section{ABSTRACT}

Sexuality and relationships are closely linked, especially in conservative societies like Mexico, where intimacy predates eroticism. Given the sociocultural context, traditional norms launch the construction of a variety of attitudes that determine the level of restriction or permissiveness of a person to engage in sexual relations. This study provides an analysis of the attitudes and behaviors towards sexuality in 112 young Mexicans by applying the sociosexual orientation inventory $\alpha=0.774$ and the attitudes toward sexuality scale $\alpha=0.736$. The results show little openness to engage in sexual activities without a vow to stay in the relationship, though with a moderate growing permissiveness trend towards short-term relationships without affection and commitment and more focused on sexual activity. Even with the sociocultural changes in sexuality, it's clear that a traditional and conservative culture has an impact on the attitudes and behaviors of young people.

Keywords: sociosexual orientation, attitudes, sexuality, intercourse, couples.

"Recibido: 23 de noviembre, 2012 - Aceptado: 23 de marzo, 2013

Correspondencia: Rolando Díaz-Loving, Facultad de Psicología, Universidad Nacional Autónoma de México, C. P. 04510, Copilco Universidad, México. e-mail: rdiazl@unam.mx 


\section{INTRODUCCIÓN}

Las actitudes hacia la sexualidad son el conjunto organizado de creencias, opiniones, sentimientos y tendencias que evalúan y disponen de determinada forma al sujeto ante personas, objetos y situaciones, relacionadas al comportamiento sexual, identidad sexual, roles de género, orientación sexual, uso de métodos anticonceptivos, la prevención de situaciones de riesgo y desarrollo de la actividad sexual (Cerruti, 1997). Estas premisas no son innatas, se adquieren mediante diversos tipos de procesos de socialización, son más o menos persistentes y suelen depender del medio donde los individuos se desarrollan (Ayala, 1999; DíazLoving \& Draguns, 1999).

En el ambito particular de la sexualidad, las actitudes cimentadas en normas y valores predeterminados por una sociedad y cultura en particular, facilitan la formación de juicios categóricos sobre los comportamientos sexuales considerados correctos o incorrectos, en ocasiones, limitando o castigando las decisiones y el actuar de las personas a través de la culpa o restricción en cuanto a la sexualidad y los mitos o tabúes que suscitan temores y recelos, ya que están basados en falacias y prejuicios en contra de las manifestaciones no reproductivas de la sexualidad (Alvarez-Gayou, 2007).

Debido al estrecho vínculo que existe entre la sexualidad y las relaciones interpersonales (Weeks, 2000), las actitudes que se construyen en torno a ella pueden tener implicaciones importantes en el inicio y mantenimiento de la relación de pareja. Si una persona posee actitudes flexibles y no restringidas hacia la sexualidad, su necesidad de diversidad o novedad sexual no estaría condicionada a experimentarse dentro de una relación formal de pareja, mientras que en personas con actitudes conservadoras o restringidas, el sexo se vería como una forma de acercarse psicológica y emocionalmente a la pareja. Cabe señalar que este patrón se amplifica entre los sexos; por ejemplo, Yela (2000) menciona que los hombres tienden a separar en mayor medida los deseos y conductas sexuales de los sentimientos y comportamientos amorosos, en comparación con las mujeres. En el mismo sentido, Ortiz Robles y García Ramos (en García, 2007) señalan que las mujeres ligan su vida erótica a lo afectivo y le dan más importancia al vínculo amoroso que a la actividad sexo-genital, a diferencia de los hombres quienes preponderan la necesidad biológica y niegan sus sentimientos.

Para explicar estas variaciones en el comportamiento sexual de los individuos, Simpson y Gangestad (1991) acuñan el constructo de orientación sociosexual que conceptualmente se entiende como el nivel de cercanía y compromiso o vínculos emocionales que se requieren para involucrarse en relaciones sexuales, lo que genera una tendencia en el comportamiento sexual personal. Una persona con orientación sociosexual restringida requiere de dichos vínculos emocionales para acceder al sexo, tiene menos parejas sexuales y menos relaciones sexuales de una sola vez; por otro lado, quienes poseen una orientación no restringida no requieren de intimidad ni compromiso para tener sexo con alguien, tienen más parejas sexuales y en muchas ocasiones tienen sexo de una sola vez (García, 2007). De esta manera, con base en los descubrimientos de Simpson y Gangestad (1991), parece que los individuos restringidos por lo general se involucran en relaciones de larga duración que se caracterizan por un mayor compromiso y lazos emocionales más fuertes, mientras que los no restringidos tienden a involucrarse en relaciones a corto plazo, caracterizadas por menos compromiso y lazos afectivos más débiles.

Ahora bien, al introducir el factor cultural, las actitudes y los comportamientos sociosexuales pueden variar aún más. La situación en cuanto a equidad de género y desarrollo económico puede predecir, en muchos países, las diferencias en cuanto a la sociosexualidad, haciendo que los parámetros del nivel sociosexual tiendan a variar de país a país (Lippa, 2009). También, en ciertas regiones que han sido afectadas históricamente por niveles altos de enfermedades infecciosas, las personas reportan niveles de sociosexualidad bajos (Schaller \& Murray, 2008).

En particular aspectos como el apego, la interdependencia y la cercanía aumentan la aceptación de la actividad sexual entre los individuos (Delamater, 1981), lo cual se manifiesta en especial en culturas latinoamericanas, donde los vínculos afectivos son necesarios para la actividad sexual (Giraldo, 2002). En México, una sociocultura predominantemente colectivista, el sexo representa una más de las expresiones de afecto, cercanía e intimidad emocional dentro de las relaciones interpersonales (García, 2007). Con base en lo anterior, 
el presente estudio pretende analizar las actitudes y comportamientos que manifiestan los jóvenes mexicanos hacia la sexualidad. Aunque los datos arrojados por esta investigación ayudarán a comprender algunos factores que intervienen en el proceso de emparejamiento y actividad sexual de los jóvenes mexicanos, no podrán ser generalizables debido al método utilizado para la selección de la muestra y al tamaño reducido de la misma.

\section{MÉTODO}

\section{Participantes}

A través de un muestreo no probabilístico por cuotas se conformó una muestra de 112 jóvenes, 62 hombres y 50 mujeres, que participaron de manera voluntaria, de entre 19 y 26 años de edad que ya tuvieron su debut sexual. La media de edad fue de 21.5 años y $89.2 \%$ de los jóvenes reportaron ser estudiantes.

\section{Instrumentos}

Escala de actitudes hacia la sexualidad (Honold, 2005)

Cuenta con un total de 24 reactivos con cuatro opciones de respuesta en una escala tipo Likert que van desde 1 , "totalmente de acuerdo", hasta 4, "totalmente en desacuerdo".

Tabla 1. Conceptuación y consistencia interna de los factores de la subescala de actitudes hacia la sexualidad

Actitudes hacia la sexualidad

$\alpha=.736$

\begin{tabular}{l|l|c}
\hline \multicolumn{1}{c}{ Actitudes hacia la sexualidad } & \multicolumn{1}{c}{ Definición } \\
\hline Factor & \multicolumn{1}{c}{$\alpha=.736$} & Alfa \\
\hline Sexismo y estereotipos & Actitudes manifestadas hacia las diferencias y estereotipos de género. & .750 \\
\hline Moralidad & $\begin{array}{l}\text { Actitudes que un individuo manifiesta respecto a lo que considera correcto } \\
\text { e incorrecto sobre el comportamiento sexual. }\end{array}$ & .670 \\
\hline Sexualidad restringida & $\begin{array}{l}\text { Actitud en la que las creencias determinan la importancia de establecer } \\
\text { vínculos emocionales para acceder al sexo. }\end{array}$ & .489 \\
\hline Mitos y tabúes & Actitudes basadas en los mitos y tabúes más comunes de la sexualidad. & .544 \\
\hline
\end{tabular}

Para adaptar y conocer las características psicométricas de la misma, se realizó un análisis factorial con rotación ortogonal (varimax) del que se obtuvieron cuatro factores (tabla 1). El punto de corte para incluir cada reactivo en un factor fue de .40; las cargas factoriales de los reactivos oscilaron entre .45 y .86 . A pesar de que en el factor de sexualidad restringida se observa un valor bajo (.489), pues contiene la menor cantidad de reactivos cuyos pesos factoriales alcanzan el valor de .83 , se determinó incluirlo una vez confirmada la congruencia conceptual entre ellos (que hicieran referencia a las creencias que determinan la importancia de los vínculos emocionales para acceder al sexo). La confiabilidad por consistencia interna para la escala completa, obtenida a través del alfa de Cronbach, fue de .736 y explica un $72.876 \%$ de la varianza total.

Inventario de Orientación Sociosexual (Simpson \& Gangestad, 1991)

Validado para población mexicana (Díaz-Loving \& García, 2008). Consta de siete reactivos: tres preguntas abiertas acerca del número de parejas sexuales pasadas y esperadas (factor conductual) y cuatro afirmaciones (factor actitudinal) que se responden con una escala tipo Likert de nueve niveles, desde "totalmente en desacuerdo" hasta "totalmente de acuerdo". Los puntajes altos en esta escala indican una menor 
necesidad de cercanía y compromiso para acceder al sexo, mientras que los bajos indican una mayor necesidad de dichos vínculos previos al sexo.

Se realizó un análisis factorial con rotación ortogonal (varimax) del que se obtuvieron dos factores (tabla 2). El punto de corte para incluir cada reactivo fue de .40; las cargas factoriales de los reactivos oscilaron entre .56 y .89 , lo que indica una consistencia interna adecuada. La confiabilidad por consistencia interna del inventario completo obtenida a través del alfa de Cronbach fue de .774 y explica un 61.83 de la varianza total.

\section{PROCEDIMIENTO}

Se llevó a cabo la aplicación individual de los instrumentos en Ciudad Universitaria, en plazas y parques de la Ciudad de México. Cada aplicador se identificó y explicó a los jóvenes el propósito de la investigación, asegurándoles la confidencialidad y anonimato. Se enfatizó la importancia de contestar con sinceridad a la totalidad de las afirmaciones. El tiempo de aplicación fue de 15 a 20 minutos. Aquellos dispuestos a colaborar debían cubrir los criterios de inclusión, o sea, haber tenido relaciones sexuales y tener entre 19 y 26 años de edad.

Una vez recolectada la información se procedió a la captura de los datos y el análisis de estadísticos descriptivos e inferenciales; con la finalidad de identificar diferencias entre los sexos se aplicó la prueba t de Student. El análisis de correlación producto momento de Pearson se utilizó para encontrar las relaciones entre las actitudes hacia la sexualidad y la orientación sociosexual. El tratamiento de los datos se realizó en el programa spss versión 17.0.

\section{RESULTADOS}

\section{Actitudes hacia la sexualidad}

En la tabla 3 se observa que las actitudes que presentan puntajes más altos son las que manifiestan mayor restricción en cuanto a la sexualidad $(\bar{X}=2.32)$, seguidas por el factor de las actitudes que revelan un alto contenido de mitos y tabúes $(\bar{X}=1.88)$. Además, se encuentran niveles moderados de moralidad $(\bar{X}=1.26)$ y sexismo $(\bar{X}=1.26)$ en las actitudes hacia la sexualidad de los jóvenes.

Tabla 2. Conceptuación y consistencia interna del Inventario de Orientación Sociosexual

\begin{tabular}{l|l|c}
\hline \multicolumn{1}{c}{ Inventario de Orientación Sociosexual } & \multicolumn{1}{c}{ Definición } & Alfa \\
\hline \multicolumn{1}{c|}{ Factor } & & .774 \\
\hline $\begin{array}{l}\text { Orientación sociosexual } \\
\text { conductual }\end{array}$ & Se refiere a las parejas sexuales pasadas, presentes y futuras. & .767 \\
\hline $\begin{array}{l}\text { Orientación sociosexual } \\
\text { actitudinal }\end{array}$ & $\begin{array}{l}\text { Se refiere a una actitud abierta y permisiva ante el sexo sin amor, } \\
\text { ante el sexo casual y ante el sexo fuera de la relación de pareja. }\end{array}$ & .780 \\
\hline
\end{tabular}

Tabla 3. Actitudes hacia la sexualidad

\begin{tabular}{l|c|c|c}
\hline & Rango & Media & Desviación estándar \\
\hline Sexismo y estereotipos & 3 & 1.29 & .495 \\
\hline Moralidad & 3 & 1.26 & .450 \\
\hline Sexualidad restringida & 3 & 2.32 & .695 \\
\hline Mitos y tabúes & 3 & 1.88 & .591 \\
\hline
\end{tabular}


Posteriormente se utilizó la prueba t de Student para muestras independientes con la finalidad de identificar si existen diferencias entre hombres y mujeres. En la tabla 4 se observa que sí hay diferencias estadísticamente significativas tanto en actitudes moralistas $(t=-2.57, p=.01)$, como en actitudes de sexismo y estereotipos ( $t=3.37, p=.001)$. En cuanto a las primeras se puede observar que las mujeres tienden más a evaluar ciertos comportamientos sexuales como incorrectos ( $\bar{X}=1.39)$ que los hombres $(\bar{X}=1.16)$. Por su parte, los hombres manifiestan actitudes más sexistas y basadas en estereotipos ( $\bar{X}=1.42$ ) que las mujeres ( $\bar{X}=1.13$ ).

No existen diferencias significativas en lo que se refiere a las actitudes sexuales restringidas y las que se basan en mitos y tabúes.

\section{Orientación sociosexual}

Con respecto al nivel de orientación sociosexual, en la tabla 5 se observa que según la media de parejas sexuales que reportan haber tenido los participantes, consi- derando las relaciones presentes, pasadas y las que se esperan en el futuro $(\bar{X}=3.05)$, los niveles conductuales de sociosexualidad se inclinan marcadamente hacia la restricción, mientras que la media del factor actitudinal de la orientación sociosexual $(\bar{X}=5.89)$ indica una tendencia moderada hacia la permisividad y apertura a involucrarse en relaciones sexuales fuera de una relación de pareja cercana y comprometida.

Posteriormente, mediante una prueba t de Student para muestras independientes, se identificaron diferencias significativas (tabla 6) entre hombres y mujeres en cuanto a los niveles de sociosexualidad actitudinal $(\mathrm{t}=4.63, \mathrm{p}=.00)$ y conductual $(\mathrm{t}=2.23, \mathrm{p}=.03)$. Los resultados obtenidos muestran que los hombres son más permisivos y abiertos a tener experiencias sexuales ocasionales y no requieren de lazos afectivos o compromiso para involucrarse en una relación sexual $(\bar{X}=5.18)$ en comparación con las mujeres ( $\bar{X}=3.51)$. Asimismo, reportan haber tenido más parejas sexuales $(\bar{X}=5.45)$ que las mujeres ( $\bar{X}=2.05)$.

Tabla 4. Diferencias en las actitudes hacia la sexualidad

\begin{tabular}{l|c|c|c|c|c}
\hline \multirow{2}{*}{ Actitudes } & \multicolumn{2}{c|}{ Hombres } & \multicolumn{2}{c}{ Mujeres } & \multicolumn{2}{c}{} \\
\cline { 2 - 6 } & \multicolumn{2}{|c|}{ Media } & T & gl & p \\
\hline Sexismo y estereotipos & 1.42 & 1.13 & 3.37 & 110 & $.00^{* *}$ \\
\hline Moralidad & 1.16 & 1.39 & -2.57 & 110 & $.01^{* *}$ \\
\hline Sexualidad restringida & 2.22 & 2.44 & -1.61 & 110 & .11 \\
\hline Mitos y tabúes & 1.94 & 1.81 & 1.15 & 110 & .25 \\
\hline
\end{tabular}

${ }^{*} p \leq .05 * * p \leq .01$

Tabla 5. Orientación sociosexual

\begin{tabular}{l|c|c|c}
\cline { 2 - 4 } & Rango & Media & $\begin{array}{c}\text { Desviación } \\
\text { estándar }\end{array}$ \\
\hline Conductual & 12 & 3.05 & 2.879 \\
\hline Actitudinal & 8 & 5.89 & 2.734 \\
\hline
\end{tabular}

Tabla 6. Diferencia por sexos en la orientación sociosexual

\begin{tabular}{|c|c|c|c|c|c|}
\hline \multirow{2}{*}{ Sociosexualidad } & Hombres & Mujeres & \multirow[b]{2}{*}{$t$} & \multirow[b]{2}{*}{ gl } & \multirow[b]{2}{*}{$p$} \\
\hline & \multicolumn{2}{|c|}{ Media } & & & \\
\hline Actitud sociosexual & 5.18 & 3.51 & 4.63 & 110 & $.001 * *$ \\
\hline Conducta sociosexual & 5.45 & 2.05 & 2.23 & 110 & $.03 *$ \\
\hline
\end{tabular}

$* p \leq .05 * * p \leq .01$ 
Correlación entre orientación sociosexual y actitudes hacia la sexualidad

Con la finalidad de conocer la relación que existe entre la orientación sociosexual y las actitudes hacia la sexualidad, se realizó una correlación producto-momento de Pearson entre estos factores.

Para las mujeres, el factor conductual de la orientación sociosexual se relaciona significativamente con las actitudes hacia sexualidad restringida $(r=-.445$; $\mathrm{p} \leq .01)$ y con los mitos y tabúes $(\mathrm{r}=-.401 ; \mathrm{p} \leq .01)$. De tal manera que a más actitudes restringidas hacia la sexualidad y basadas en mitos y tabúes, menor es la cantidad de parejas sexuales que se han tenido o se esperan tener. Del mismo modo, el factor actitudinal de la orientación sociosexual se relaciona con la sexualidad restringida $(\mathrm{r}=-.607 ; \mathrm{p} \leq .01)$ y con los mitos y tabúes $(\mathrm{r}=-.602 ; \mathrm{p} \leq .01)$, es decir, mientras más permisiva es la mujer en cuanto a involucrarse en relaciones sexuales sin compromiso, manifestará menos actitudes restringidas y basadas en mitos y tabúes.
En los hombres (tabla 8), el factor actitudinal de la sociosexualidad se relaciona con las actitudes hacia la sexualidad de moralidad ( $\mathrm{r}=-.281 ; \mathrm{p} \leq .05)$, restricción $(\mathrm{r}=-.415 ; \mathrm{p} \leq .01)$ y cargadas de mitos y tabúes $(\mathrm{r}=-.349 ; \mathrm{p} \leq .01)$. Es decir, se observa que a mayor apertura y permisividad para involucrarse en relaciones sexuales sin compromiso y sin lazos emocionales, menos se manifiestan las actitudes de restricción, moralidad y aquellas basadas en mitos y tabúes.

\section{DISCUSIÓN}

La cultura es capaz de programar la mente sexual proporcionando guías de comportamiento que son internalizadas y que favorecen que las personas desarrollen un estilo de ser sexual (Levine, 2002). Las culturas colectivistas valoran la interdependecia emocional, como en la mexicana, por lo que se caracterizan por la tendencia general de vincular el sexo y el afecto (Díaz-Loving \& García, 2008). Así, el proceso de socializacion es determinante en la formacion de las actitudes hacia la sexualidad (Ayala, 1999).

Tabla 7. Relación entre actitudes hacia la sexualidad y orientación sociosexual en mujeres

\begin{tabular}{l|c|c}
\cline { 2 - 3 } & Conductual & Actitudinal \\
\hline Sexismo y estereotipos & -.061 & -.261 \\
\hline Moralidad & -.150 & .185 \\
\hline Sexualidad restringida & $-.445^{* *}$ & $-.607^{* *}$ \\
\hline Mitos y tabúes & $-.401 * *$ & $-.602 * *$ \\
\hline
\end{tabular}

${ }^{*} p \leq .05 * * p \leq .01$

Tabla 8. Relación entre actitudes hacia la sexualidad y orientación sociosexual en hombres

\begin{tabular}{l|c|c}
\cline { 2 - 3 } & Conductual & Actitudinal \\
\hline Sexismo y estereotipos & .161 & -.092 \\
\hline Moralidad & .208 & $-.281^{*}$ \\
\hline Sexualidad restringida & -.212 & $-.415^{* *}$ \\
\hline Mitos y tabúes & .147 & $-.349^{* *}$ \\
\hline
\end{tabular}

${ }^{*} p \leq .05 * * p \leq .01$ 
Dentro del marco de conservadurismo que caracteriza a los jóvenes hispanos (Eisenman \& Dantzker, 2004), resulta congruente encontrar que para los jóvenes mexicanos es importante establecer vínculos emocionales para acceder al sexo. Los hallazgos del presente estudio muestran la tendencia moderada a requerir el establecer lazos afectivos y de compromiso para involucrarse en cualquier tipo de actividad sexual (ni sumamente restringida ni muy abierta), pues el componente actitudinal de la orientación sociosexual acorde con el factor de las actitudes ante la sexualidad restringida se ubica muy cercanos a la media, cargándose ligeramente hacia las actitudes de permisividad.

En la sociedad mexicana, la estrecha interacción de la cultura y la religión (Collignon, 2011) además de las condiciones políticas, económicas y sociales, limitan y problematizan el ejercicio del erotismo generando tensiones y contradicciones (García, 2007). A pesar de que el nivel de las actitudes sociosexuales tiende ligeramente a la permisividad, se observa una clara restricción del comportamiento sexual en el componente conductual de la orientación sociosexual que evalúa la cantidad de parejas sexuales que se han tenido en el presente, pasado y que se esperan en un futuro. Dicho contraste entre las actitudes y las conductas enfatiza las tensiones al interior de los individuos y entre las personas con relación a los deseos, las emociones, las expectativas y las experiencias sexuales (García, 2007).

Este estudio revela resultados que complementan los hallazgos reportados en García (2007) y DíazLoving y García (2008), pues en sus estudios sobre el comportamiento sexual y la sociosexualidad de adultos mexicanos, ambos componentes (actitudinal y conductual) reflejan una orientación sociosexual restringida o poco abierta. Resultados similares se desprenden de la presente investigación, aunque varían en cuanto al componente conductual de la sociosexualidad, que refleja una ligera tendencia a la permisividad, probablemente debido a que la muestra de este estudio estuvo conformada por gente más joven que en los estudios que le preceden.

En cuanto a las diferencias entre los sexos, se encontró que el nivel de orientación sociosexual de los hombres es significativamente mayor al de las mujeres; ellos presentan actitudes más abiertas y permisivas con respecto a las actividades sexuales sin compromiso y tienen un mayor número de parejas sexuales en comparación con las mujeres. Además, requieren menos niveles de intimidad y compromiso para tener sexo, lo cual los lleva a tener más parejas sexuales a lo largo de su vida. Son más abiertos y menos restringidos que las mujeres tanto en actitudes como en conducta (DíazLoving \& García, 2008).

Este estudio también determina la correlación estadísticamente significativa que existe entre la sociosexualidad y las actitudes ante la sexualidad. Particularmente en el grupo de las mujeres, donde se encontraron correlaciones más fuertes, aquellas que manifiestan actitudes restringidas y basadas en mitos y tabúes son menos permisivas o menos abiertas en cuanto al sexo sin compromiso o sexo de una sola vez; además, reportan haber tenido menos parejas sexuales. Lo mismo se da en dirección opuesta: las mujeres que reportaron haber tenido más parejas sexuales o estar más dispuestas al sexo casual o sin compromiso revelan actitudes menos restringidas y creen menos en los mitos y tabúes que normalmente envuelven a la sexualidad.

El proceso de socialización diferencial para hombres y mujeres es palpable a pesar de los intentos por conformar una sociedad más igualitaria. Aunque existe una continua modificación de los roles con las transformaciones de carácter sociocultural, la predominancia de actitudes sexistas en los hombres y de la moralidad de las mujeres, encontrados en esta investigación, refleja que algunos aspectos vinculados a creencias que perduran por la fuerza de las costumbres no necesariamente cambian al compás de la evolución social (Rocha \& Díaz-Loving, 2011). De tal forma que los hombres que manifiestan actitudes restringidas que además obedecen a las normas morales establecidas en sociedad sobre lo que una mujer y un hombre deben o no hacer, o actitudes que se producen a partir del conocimiento de ciertos mitos o tabúes que rodean a la sexualidad, tienden a expresar actitudes menos permisivas o abiertas en cuanto al sexo sin compromiso, o idealmente piensan que crear lazos emocionales y compromiso es importante para involucrarse en una relación sexual.

Por otro lado, los hombres han sido socializados centrados en el placer (Delamater, 1987) en el proceso de la construcción de su identidad, donde la esfera sexual representa un reflejo de su hombría, por lo que desarrollan una serie de conductas sexuales que res- 
ponden a lo que se espera socialmente de su rol masculino (Rocha \& Díaz-Loving, 2011). Así, encontramos que el número de parejas que los hombres tuvieron en el pasado, presente o esperan tener en el futuro no varía en función de las actitudes que puedan manifestar hacia la sexualidad, independientemente del nivel en que estas actitudes sean restringidas, sexistas, moralistas o basadas en mitos, por lo que es razonable encontrar que sus comportamientos sean menos restringidos que los de las mujeres, a quienes se enseña a reprimir sus deseos y rechazar el sexo para preservar la virtud (Baumeister, 2001) a través de normas culturales que históricamente han reprimido su sexualidad (Amuchástegui, 1998).

Durante la juventud, los rasgos y comportamientos que distinguen a los roles de género emprenden un proceso de ajuste donde finalmente se espera que las personas se apeguen marcadamente a lo largo de su desarrollo a dichos roles masculinos o femeninos (DíazLoving \& Rocha, 2008). Las diferencias en actitudes que se detectaron dentro de los factores de sexismoestereotipos y moralidad, donde las mujeres manifiestan mas actitudes moralistas y los hombres más actitudes sexistas, aluden a la posibilidad de que los individuos agrupan la información que obtienen de manera que sea congruente con su identidad de género, desarrollando actitudes que suelen responder a patrones socioculturales normativos (Díaz-Loving \& Rocha, 2008).

Como corolario, se observa que la tendencia hacia actitudes y conductas restringidas es común en las sociedades colectivistas, como la cultura mexicana, donde se valora la dependencia emocional y se regulan las prácticas sexuales en torno a la relación de pareja (Díaz-Loving \& García, 2008). Es así que la socialización tradicionalista promueve, en los jóvenes mexicanos, actitudes dirigidas a valorar las características de afecto y protección que suelen favorecer las relaciones de larga duración, donde la exploración del erotismo constituye un intento de alcanzar intimidad y compromiso dentro de sus interacciones con el otro, tal como el nivel de orientación sociosexual nos permite entender en la presente investigación, los jóvenes que poseen una orientación restringida prefieren y elijen personas de atributos personales relacionados con la estabilidad y el compromiso como la lealtad, compatibilidad y capacidad afectiva (Simpson \& Gangestad, 1992). Aun así, los jóvenes comienzan a manifestar actitudes que tienden a la permisividad y apertura para establecer relaciones a corto plazo disociadas del afecto y compromiso enfocadas en la actividad sexual, aunque las implicaciones de un estigma social que conlleva actuar de acuerdo con esta permisividad pueden estar restringiendo ciertos comportamientos sexuales. 


\section{REFERENCIAS}

Amuchástegui, A. (1998). Saber o no saber sobre sexo: los dilemas de la actividad sexual femenina para jóvenes mexicanos. En I. Szasz \& S. Lerner (comps.), Sexualidades en México, Algunas aproximaciones desde la perspectiva de las ciencias sociales. México: El Colegio de México.

Alvarez-Gayou, J. (2007). Educación de la sexualidad: ¿en la casa o en la escuela? Los géneros, la escuela y la educación profesional de la sexualidad. México: Paidós.

Ayala, M. (1999). Actitudes que presentan los padres y madres de adolescentes acerca de que se les imparta educación de la sexualidad a sus hijos e hijas en la escuela. Archivos hispanoamericanos de sexología. 5(2), 165-183.

Baumeister, R. F. (2001). Social Psychology and Human Sexuality. Essential readings. UsA: Taylor and Francis Group.

Cerruti, S. (1997). Educación de la sexualidad en el contexto de la salud integral en la adolescencia. Organización Panamericana de la Salud. Fundación W. K. Kellogg. Montevideo: OPS-OMS.

Collignon Goribar, M. (2011). Discursos sociales sobre la sexualidad: narrativas sobre la diversidad sexual y prácticas de resistencia. Comunicación y Sociedad, 133-160.

Delamater, J. (1981). The Social Control of Sexuality. Annual Review of Sociology, 7(1), 263-290.

Delamater, J. (1987). Gender differences in sexual scenarios. En Kelly (Ed.) Females, males and sexuality (pp. 127-139). UsA: State University of New York Press.

Díaz-Loving, R. y Draguns, J. (1999). Socioculture. Meaning and Personality in Mexico and in the United States. En Y. T. Lee, C. McCauley y J. Draguns (Eds.) Personality and person perception across cultures. (pp. 103-126). New Jersey, London: Lawrence Erlbaum Associates Publishers.

Díaz-Loving, R. \& García, G. (2008). Sociosexual Orientation and Sexual Behavior in Mexican Adults. Social and Personality Psychology Compass 2(3), 1199-1217.

Diaz-Loving, R. \& Rocha, T. (2008). La masculinidad y la feminidad a través del ciclo vital: un estudio comparativo. La psicología social en México. 12, 215-226.
Eisenman, R. \& Dantzker, M. L. (2004). Sex Differences in Sex Attitudes Among Hispanic College Students. International Journal of Social Rehabilitation, 9(17-21).

García, G. (2007). Conducta sexual: un modelo psicosocial. (Tesis doctoral). Facultad de Psicología. UNAM, México.

Giraldo, O. (2002). Nuestras sexualidades. Colombia: Litocencoa.

Honold, J. (2005). Diseño de un instrumento para la evaluación de información y actitudes ante la sexualidad. (Tesis de Licenciatura). unAm, México.

Levine, S. B. (2002). Reexploring the concept of sexual desire. Journal of sex and marital therapy, 28, 39-51.

Lippa, R. A. (2009). Sex differences in sex drive, sociosexuality, and height across 53 nations: testing evolutionary and social structural theories. Archives of Sexual Behavior. 38(5): 631-51.

Ortiz Robles, R. y García Ramos, J. C. (2002). La influencia de la cultura en la satisfacción sexual del adulto maduro. En http://www.uaq.mx/psicologia/lamision/ influencia.html Accesado el 13 de agosto de 2005.

Rocha, T. \& Díaz-Loving, R. (2011). Identidades de género. Más allá de cuerpos y mitos. México: Trillas.

Schaller, M. \& Murray, D. R. (2008). Pathogens, personality, and culture: Disease prevalence predicts worldwide variability in sociosexuality, extraversion, and openness to experience. Journal of Personality and Social Psychology, 95(1), 212-221.

Simpson, J. \& Gangestad, S. (1991). Individual differences in sociosexuality: evidence of convergent and discriminant validity. Journal of personality and social psychology. 60(6), 870-882.

Simpson, J. \& Gangestad, S. (1992). Sociosexuality and romantic partner choice. Journal of Personality. 60, 31-51.

Weeks, J. (2000). Los valores sexuales en los tiempos del SIDA. En Szasz, I. y Lerner, S. (Comps.) Sexualidades en México. Algunas aproximaciones desde la perspectiva de las ciencias sociales. México: El Colegio de México.

Yela, C. (2000). Predictors of and factors related to loving and sexual satisfaction for men and women. European review of applied psychology. 42, 235-242. 